\title{
Great occipital nerve long-acting steroid injections in cluster headache therapy: an observational prospective study
}

\author{
Elena Merli ${ }^{1}$. Gian Maria Asioli ${ }^{1}$. Valentina Favoni ${ }^{1}$ - Corrado Zenesini $^{1} \cdot$ Davide Mascarella $^{1} \cdot$ Alex Sartori $^{1}$. \\ Pietro Cortelli ${ }^{1,2} \cdot$ Sabina Cevoli ${ }^{1}$ (I) $\cdot$ Giulia Pierangeli ${ }^{1,2}$
}

Received: 16 September 2021 / Revised: 30 October 2021 / Accepted: 1 November 2021 / Published online: 25 November 2021

(c) The Author(s) 2021

\begin{abstract}
Background Injections targeting the occipital nerve are used to reduce headache attacks and abort cluster bouts in cluster headache patients. There is no widely accepted agreement over the optimal technique of injection, type and doses of steroids and/or anesthetics to use, as well as injection regimens. The aim of this study was to verify the effectiveness and safety of greater occipital nerve long-acting steroid injections in the management of episodic and chronic cluster headache.

Methods We conducted a prospective observational cohort study on episodic $(\mathrm{ECH})$ and chronic cluster headache patients $(\mathrm{CCH})$. ECH were included in the study at the beginning of a cluster period. Three injections with $60 \mathrm{mg}$ methylprednisolone were performed on alternate days. We registered the frequency and intensity of attacks three days before and 3, 7 and 30 days after the treatment, the latency of cluster relapse, adverse events, scores evaluating anxiety (Zung scale), depression (Beck's Depression Scale) and quality of life (Disability Assessment Schedule II, 12-Item Self-Administered Version). Primary outcome was the interruption of the cluster after the three injections. Responders conducted a follow-up period of 12 months. Results We enrolled 60 patients, 47 with ECH and 13 with CCH. We observed a complete response in $47.8 \%(22 / 46)$ of episodic and 33.3\% (4/12) of chronic patients. Moreover, a partial response (reduction of at least $50 \%$ of attacks) was obtained in further $10.8 \%$ (5/46) of episodic and in 33.3\% (4/12) of chronic patients at 1 month. Median pain-free period was of 3 months for $\mathrm{CCH}$ responders. Only mild adverse events were reported in $38.3 \%$ (23/58) cases.

Conclusions We suggest three greater occipital nerve injections of $60 \mathrm{mg}$ methylprednisolone on alternate days as useful therapy in episodic and chronic cluster headache. This leads to a long pain-free period in chronic forms. Adverse effects are mild and support its use as first choice.
\end{abstract}

Trial registration The study was inserted in AIFA observational studies register.

Keywords Cluster headache $\cdot$ Transitional therapy $\cdot$ Great occipital nerve $\cdot$ Methylprednisolone

$\begin{array}{ll}\text { Abbreviations } \\ \text { CH } & \text { Cluster headache } \\ \text { ECH } & \text { Episodic cluster headache } \\ \text { CCH } & \text { Chronic cluster headache } \\ \text { GON } & \text { Great occipital nerve } \\ \text { CGRP } & \text { Calcitonin gene-related peptide }\end{array}$

Sabina Cevoli

sabina.cevoli@unibo.it

1 IRCCS Istituto delle Scienze Neurologiche di Bologna, Ospedale Bellaria, via Altura 3, Bologna, Italy

2 DIBINEM, Department of Biomedical and Neuromotor Sciences, University of Bologna, Bologna, Italy

\section{Introduction}

Cluster headache $(\mathrm{CH})$ is among the most painful headache disorders. An attack of severe, unilateral, orbital or temporal pain, along with ipsilateral cranial autonomic features and a sense of restlessness may occur up to 8 times a day and last from 15 to $180 \mathrm{~min}$ [1]. In episodic $\mathrm{CH}(\mathrm{ECH})$, attacks occur in series lasting for weeks or months (so-called cluster periods) separated by remission periods usually lasting months or years. In $10-15 \%$ of patients, $\mathrm{CH}$ begins as or evolves into a chronic form (chronic $\mathrm{CH}, \mathrm{CCH}$ ), in which remission periods last less than three months or are completely absent [1]. Medical treatment of $\mathrm{CH}$ includes acute, transitional and preventive therapy. Acute treatment aims to abort the pain of each attack, while preventive therapy modulates attack frequency, intensity and cluster duration. Oral preventive 
treatments, such as verapamil, lithium or valproate, frequently cause side effects and need days or weeks to be active. Transitional therapy should provide almost immediate relief to decrease pain until preventive therapy becomes effective or until the cluster period ends spontaneously [2]. Although transitional therapy commonly consists of oral corticosteroids [3], injections targeting the greater occipital nerve $(\mathrm{GON})$ are a local alternative to reduce headache attacks and abort cluster bouts [4].

Patterns of use of GON injections greatly vary, as there is no widely accepted agreement among headache specialists over the optimal injection regimens, type and dose of steroids and/or anesthetics [5].

The most numerous randomized placebo-controlled trial indicates a protocol of three repeated steroid injections on alternate days [6]. Drawing inspiration from that protocol, we selected methylprednisolone which is a low-cost and easily available medication and whose use is supported by most previous studies on GON injections.

Therefore, we hypothesized that repeated injections of long-acting steroid could be useful in $\mathrm{CH}$ treatment. The aim of our study was to verify the effectiveness and safety of GON long-acting steroid injections alone in the management of $\mathrm{ECH}$ and $\mathrm{CCH}$ patients.

\section{Materials and methods}

This is an observational prospective cohort study. We consecutively recruited all patients with $\mathrm{ECH}$ and $\mathrm{CCH}$ attending the Headache Center of IRCSS-Istituto delle Scienze Neurologiche di Bologna, from October 2017 to October 2019 and fulfilling inclusion criteria. Inclusion criteria were: subjects over the age of 18; diagnosis of ECH and $\mathrm{CCH}$ according to the criteria of "The international classification of headache disorders" ICHD Edition 3-beta [1]; ECH in active cluster within the first week from cluster onset and $\mathrm{CCH}$ during the exacerbation phase; if taking preventive therapies, these should have not been modified in the previous 3 months. As ICHD3 diagnostic criteria for cluster headache were published after the study initiation, we maintained the interim ICHD3-beta criteria throughout the study. Exclusion criteria were: patients with another type of headache; patients with non-active cluster headache; patients with contraindications to methylprednisolone; patients taking anticoagulants or with coagulation disorders; patients using oral steroid therapy; patients with preventive therapies introduced or modified in the previous 3 months; patients unable to sign the informed consent. The protocol consisted in three injections on alternate days. Each injection was performed on the side of the pain using a syringe with $60 \mathrm{mg}$ of methylprednisolone solved in $2 \mathrm{ml}$ of saline solution, with a 25 -gauge needle. GON was localized by presuming a line from the occipital protuberance to the mastoid process and moving $1 / 3$ of the way laterally as previously reported [7]. The insertion was performed $2 \mathrm{~cm}$ below the occipital nuchal ridge, carefully aspirating before injecting, to ensure that the needle was not inside a vessel.

Visits occurred at baseline (preliminary visit T0), 3 days after baseline (T1), 2 and 4 days after T1 (T2 and T3), 1 month after treatment (T4), then at 3 months (T5), 6 months (T6) and 12 months (T7) after the end of treatment.

At $\mathrm{T} 0$, while recruiting the patient, inclusion and exclusion criteria were verified and all information regarding participation in the study and treatment of personal data was discussed. A free informed consent form was signed. Previous clinical history was traced (age of onset of $\mathrm{CH}$, duration of previous clusters, characteristics of pain, frequency and intensity of attacks, previous and concomitant preventive therapies, comorbidities, lifestyle). Patients were asked to daily fill in a headache diary, detailing the number of attacks, rating pain severity using the Numerical Rating Scale, and assessing acute therapies used. Diaries were recollected at each follow-up visit. The infiltrations were performed at T1, $\mathrm{T} 2$ and T3. One month after the last injection (T4) we asked patients to return their headache diary, and asked patients to complete questionnaires. The complete responders group had also a follow-up visit at 3, 6 and 12 months to verify the relapse of the cluster. At each visit they filled in tests regarding anxiety (Zung's scale) [8], depression (Beck's Depression Scale) [9] and quality of life (Disability Assessment Schedule II, 12-Item Self-Administered Version) [10].

\section{Outcome measures}

Primary outcome was to evaluate the number of complete responders, i.e. those with complete disappearance of attacks after the third infiltration and who maintained the efficacy at least for the first month. Secondary outcomes were to evaluate the number of partial responders, i.e., those with an improvement of at least $50 \%$ in the frequency of attacks after one month from treatment compared to previous frequency, the reduction of the intensity of pain, the evaluation of recurrence of cluster attacks at one-year of follow-up, the improvement of anxiety, depression and quality of life. We considered a no response as the absence of any significant therapeutic effect.

\section{Standard protocol approvals and patient consents}

The study was approved by the Local Ethics Committee of the health service of Bologna (Comitato Etico Indipendente Area Vasta Emilia Centro: CE 17130). All patients gave their written informed consent to study participation. 


\section{Statistical analysis}

Continuous variables were presented as mean and standard deviation (SD) or median and interquartile range (IQR), the normality of distribution was evaluated with Shapiro-Wil test. Categorical variables were presented as absolute $(n)$ and relative frequency (\%). Analyses were performed for all patients and for $\mathrm{ECH}$ and $\mathrm{CCH}$ patients sub-groups.

The primary and secondary outcomes were presented as absolute $(n)$ and relative frequencies $(\%)$. Wilcoxon matched pairs signed-ranks test was used to compare pain intensity and number of attacks over the time at 3, 7 and 30 days after the baseline. Wilcoxon matched pairs signed-ranks test was used to compare Zung's scale, Beck's Depression Scale and Disability Assessment Schedule II scores at 30 months compared to the baseline. The results were presented as median reduction and interquartile range reduction compared to the baseline. Finally, we used Kruskal-Wallis test to compare the variations of the scales above and duration of illness between the three groups: responders, partial and not responders. Two-sided $p$ values were presented.

Statistical analysis was performed using statistical package Stata SE, 14.2.

\section{Results}

Sixty $\mathrm{CH}$ patients were enrolled: $47 \mathrm{ECH}$ and $13 \mathrm{CCH}$. Demographic and baseline characteristics are included in Table 1 . Oral preventive therapy was ongoing in 10 out of $13 \mathrm{CCH}$ patients $(76.9 \%)$ and in 18 out of $47 \mathrm{ECH}$ patients $(38.3 \%)$. One ECH discontinued the study after the first
Table 1 Description of $\mathrm{CH}$ patients at baseline

\begin{tabular}{|c|c|c|c|}
\hline & $\begin{array}{l}\text { Total CH } \\
N=60\end{array}$ & $\begin{array}{l}\mathrm{CCH} \\
N=13\end{array}$ & $\begin{array}{l}\mathrm{ECH} \\
N=47\end{array}$ \\
\hline \multicolumn{4}{|l|}{ Sex } \\
\hline $\mathrm{M}-n(\%)$ & $50(83.3)$ & $11(84.6)$ & $39(83.0)$ \\
\hline $\mathrm{F}-n(\%)$ & $10(16.7)$ & $2(15.4)$ & $8(17.0)$ \\
\hline Age-mean (SD) & $44(11.9)$ & $46.1(15.6)$ & $43.7(10.8)$ \\
\hline Age of onset-median (IQR) & $27(20-31)$ & $34(25-40)$ & $25(19-30)$ \\
\hline Duration of illness $(y)$-mean & 16.6 & 11.8 & 17.9 \\
\hline \multicolumn{4}{|l|}{ Smoke } \\
\hline Non-smoker- $n(\%)$ & $14(23.3)$ & $1(7.7)$ & 13 (27.6) \\
\hline Previous smoker- $n(\%)$ & $18(30)$ & $4(30.8)$ & $14(29.8)$ \\
\hline Active smoker $-n(\%)$ & $28(46.7)$ & $8(61.5)$ & $20(42.6)$ \\
\hline Number of daily attacks—-median (IQR) & $2(1-3)$ & $2(1-3)$ & $2(1-3)$ \\
\hline Intensity of attacks—median (IQR) & $8(7-9.5)$ & $8(8-9)$ & $8(7-10)$ \\
\hline Duration of $\mathrm{CH}$ since onset-median (IQR) & $16(7-23)$ & $11(5-18)$ & $17(10-26)$ \\
\hline Frequency of clusters/year-median (IQR) & - & - & $1(0.5-1)$ \\
\hline Duration of cluster (month)—median (IQR) & - & $8(5-9)$ & $1.5(1-2)$ \\
\hline \multicolumn{4}{|l|}{ For CCH only } \\
\hline Chronic at onset $-n(\%)$ & & $6(46.2)$ & \\
\hline Episodic at onset $-n(\%)$ & & $7(53.8)$ & \\
\hline \multicolumn{4}{|l|}{ Ongoing preventive therapy } \\
\hline Yes- $n(\%)$ & $28(46.6)$ & $11(84.6)$ & $17(36.1)$ \\
\hline No- $-n(\%)$ & $32(53.3)$ & $2(15.4)$ & $30(63.83)$ \\
\hline \multicolumn{4}{|l|}{ Type of preventive therapies } \\
\hline - Verapamil & & 6 & 17 \\
\hline - lithium & & 1 & - \\
\hline - Verapamil plus lithium & & 2 & 1 \\
\hline - Verapamil plus valproate & & 1 & - \\
\hline Previous oral steroid therapy $-n(\%)$ & $14(23.3)$ & $1(7.7)$ & $13(27.6)$ \\
\hline Response Yes- $n(\%)$ & $8(57.1)$ & $1(100)$ & $7(53.8)$ \\
\hline Zung's Scale score at T0-mean (SD) & $33.34(5.70)$ & $33.54(6.28)$ & $33.28(5.61)$ \\
\hline Beck's Depression Scale score at T0—mean (SD) & $10.74(8.06)$ & $10.62(8.36)$ & $10.77(8.07)$ \\
\hline WHODAS 2.0 score at T0-mean (SD) & $17.76(14.30)$ & $14.47(12.65)$ & $18.54(14.70)$ \\
\hline
\end{tabular}

$\mathrm{CH}$ cluster headache, $\mathrm{ECH}$ episodic cluster headache, $\mathrm{CCH}$ chronic cluster headache, $S D$ standard deviation, $I Q R$ interquartile range, $y$ year 
injection because of trypanophobia. One $\mathrm{CCH}$ was lost at follow-up. Among the remaining 58 patients, 26 (44.8\%) were attack free after 1 month from the third injection. Comparing $\mathrm{ECH}$ and $\mathrm{CCH}$ patients, complete responders were $22 / 46(47.8 \%)$ and $4 / 12(33.3 \%)$, respectively (Table 2). Furthermore, $5 / 46$ of ECH (10.8\%) and 4/12 of CCH (33.3\%) were partial responders reaching the secondary outcome. Hence, altogether, about $2 / 3$ of $\mathrm{CCH}$ ameliorated their frequency of attacks/day of $>50 \%$ from the baseline. We describe a slight, non-significant improvement in pain intensity throughout the month even in non-responders. Detailed results on the reduction of frequency and intensity of pain during the month are reported on Table 2. Among the analyzed variables, duration of $\mathrm{CH}$ since onset negatively correlates with a good response to treatment: responders had a short duration of $\mathrm{CH}$ (median 10 years since $\mathrm{CH}$ onset (IQR 5-18) compared to non-responders (median 20 years (IQR 11-26); $p=0.022$ ). On the contrary, previous response to oral steroid therapy, the frequency, intensity or duration of attacks, sex, smoking, and concomitant therapies did not correlate with treatment response. A proportion of 23 patients of the whole sample (38.3\%) reported mild adverse

Table 2 Results

\begin{tabular}{llll}
\hline & $\begin{array}{l}\text { Total CH } \\
(N=58) \\
n-\%\end{array}$ & $\begin{array}{l}\mathrm{CCH} \\
(N=12) \\
n-\%\end{array}$ & $\begin{array}{l}\mathrm{ECH} \\
(N=46) \\
n-\%\end{array}$ \\
\hline Complete responders $^{\mathrm{a}}$ & $26(44.8)$ & $4(33.3)$ & $22(47.8)$ \\
Partial responders $^{\mathrm{b}}$ & $9(15.5)$ & $4(33.3)$ & $5(10.8)$ \\
Median reduction of n of attacks/day $_{\text {T2 }}$ & -0.6 & -2 & -0.55 \\
T3 & -0.55 & -0.5 & -0.55 \\
T4 & -0.8 & 0 & -0.1 \\
\hline
\end{tabular}

$\mathrm{CH}$ cluster headache, $\mathrm{ECH}$ episodic cluster headache, $\mathrm{CCH}$ chronic cluster headache, $S D$ standard deviation; ${ }^{\S}$ Complete responders $=$ those with complete disappearance of attacks after the third infiltration and who maintained the efficacy at least for the first month; 'Partial responders =improvement of at least $50 \%$ in the frequency of attacks after one month from treatment compared to previous frequency events: 21 complained of neck stiffness and 2 of mild pain on the site of injection. Among them, five patients (8.3\%) still complained of neck stiffness after 1 month. No serious nor systemic adverse events were observed. Complete responders underwent a 1-year follow-up: 50\% of ECH were still pain-free at 1-year of follow-up, while $50 \%$ had recurrence of cluster attacks with a mean latency of 8 months (242 days, $\mathrm{SD} \pm 130.49)$. All $\mathrm{CCH}$ patients relapsed with a median pain-free period of 3 months (94 days, $\mathrm{SD} \pm 7.85$ ). Zung's scale, Beck's Depression Scale and Disability Assessment Schedule II at one month revealed a slight improvement compared to baseline. No significant difference was shown comparing responders to non-responders (Table 3).

\section{Discussion}

Our study shows the effectiveness and safety of GON longacting steroid injections alone as a transitional therapy in a large sample of $\mathrm{CH}$ patients. This is the first study that evaluated the use of methylprednisolone at the high dosage of $60 \mathrm{mg}$, repeated in three injections on alternate days on the side of pain. We found a high efficacy in stopping the cluster period: $45 \%$ of patients (48\% of ECH and $33 \%$ of $\mathrm{CCH}$ ) were attack free for at least one month after the third injection. Moreover, a further proportion of $11 \%$ of $\mathrm{ECH}$ and $33 \%$ of $\mathrm{CCH}$ diminished the frequency of attacks per day of at least $50 \%$, reaching the secondary outcome.

A variety of studies suggest that GON injections represent a safe and useful therapy in $\mathrm{CH}$ (see Table 4) [2, $5,6,11-18]$. In spite of this, data from literature are not easily comparable due to the differences in inclusion criteria, protocol of treatment, type and doses of steroid and/ or anesthetics used and clinical outcome observed. Most protocols provide a combination of steroid plus anesthetic injection or a simple anesthetic block, however, only a few studies can boast an adequate randomized, placebocontrolled design. In the most numerous one [6], Leroux proved the efficacy of three repeated injections on alternate days of cortivazol in ECH and CCH. Drawing inspiration
Table 3 Anxiety, depression and quality of life evaluation at Day 30 compared to Day 0

\begin{tabular}{llll}
\hline $\begin{array}{l}\text { Mean reduction } \\
- \text { mean (SD) }\end{array}$ & Zung's scale & Beck's Depression Scale & $\begin{array}{l}\text { Disability } \\
\text { assessment } \\
\text { schedule II }\end{array}$ \\
\hline Complete responders $^{\mathrm{a}}$ & $-0.23(4.7)$ & $-5.25(8.34)$ & $-9.58(14.7)$ \\
Partial responders $^{\mathrm{b}}$ & $-1.25(5.0)$ & $-2.87(2.41)$ & $-7.4(7.7)$ \\
Non-responders & $-3(4.5)$ & $-2.6(5.9)$ & $-1.0(11.6)$ \\
$P$ & 0.285 & 0.476 & 0.442 \\
\hline
\end{tabular}

$\mathrm{CH}$ cluster headache, $E C H$ episodic cluster headache, $\mathrm{CCH}$ chronic cluster headache, $S D$ standard devia-

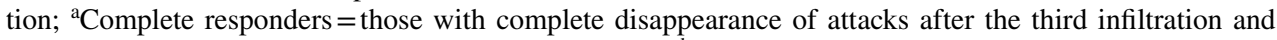
who maintained the efficacy at least for the first month; ${ }^{b}$ Partial responders =improvement of at least $50 \%$ in the frequency of attacks after one month from treatment compared to previous frequency 


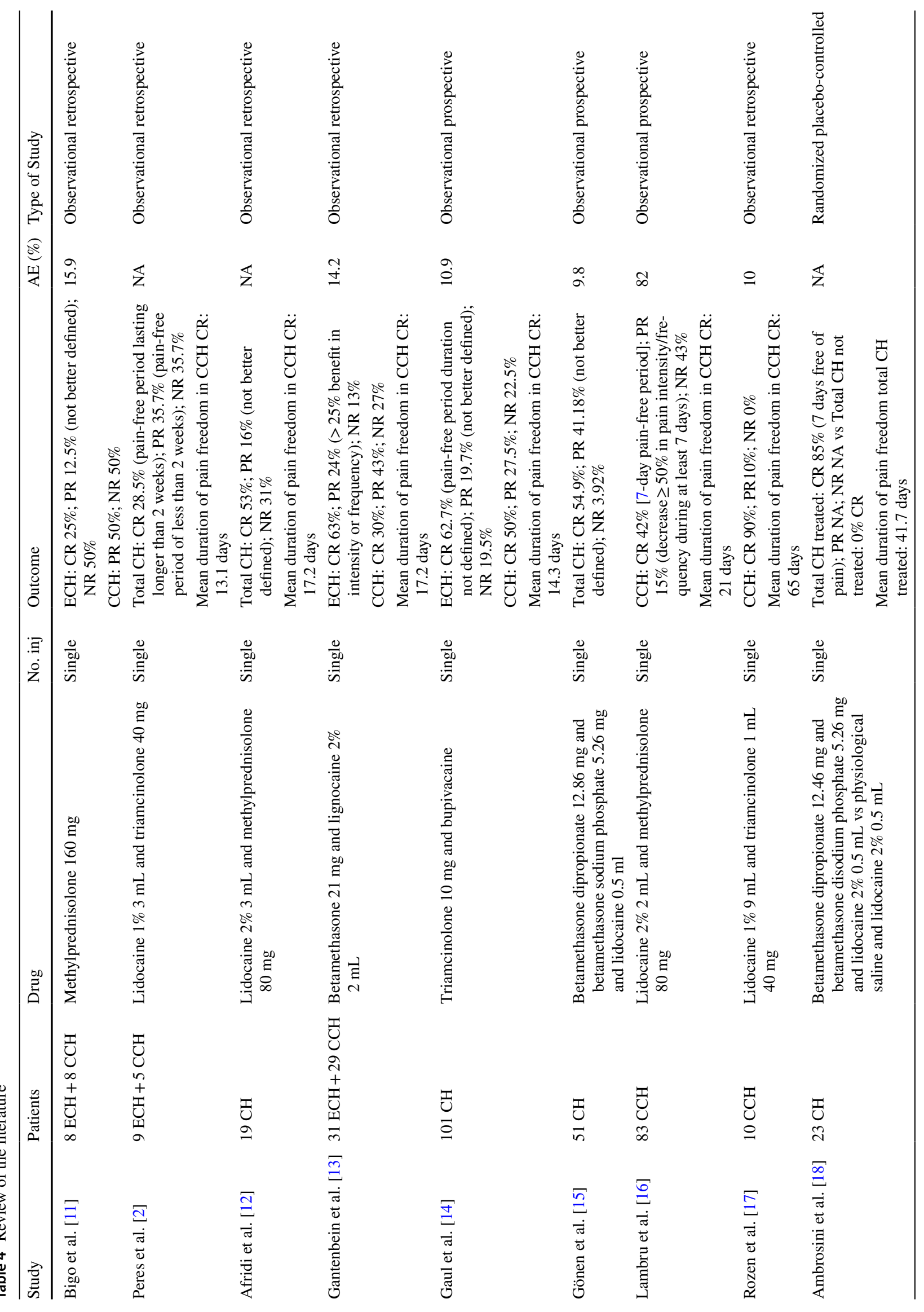


from the experience of Leroux, we selected methylprednisolone which is a low-cost and easily available medication and whose use is supported by most previous studies on GON injections. Overall, in previous studies a complete response was variably shown in 28-63\% of $\mathrm{CH}$ (25-63\% in ECH; 30-90\% in CCH) (see Table 4). Our results are in line with the ones reported.

Since comparable results were achieved in protocols with local anesthetic or steroids alone, we may suppose that a combination of anesthetics plus steroids does not produce a significantly better response. The clinical benefit is therefore most likely attributable to the steroid. Interestingly, our study demonstrates for the first time that previous response to oral steroid therapy does not correlate with that of steroid injection therapy. This supports the idea of a prevalent local mechanism of action of GON injection, which is not linked to the systemic action of the steroids. In fact, GON is traditionally thought to be a therapeutic target due to the anatomo-physiological convergence of $\mathrm{C} 2$ dermatome and trigeminovascular afferents in the spinal trigeminal nucleus, which underlie the reported pain from orbitofrontal regions [19].

The long follow-up is certainly a strong point of our study. After one-year from injection 50.0\% of ECH complete responders were still cluster free while the other half relapsed with a mean latency of 8 months. A relevant result is that $\mathrm{CCH}$ complete responders obtained a median pain-free period of 3 months, substantially longer than previously reported (13-65 days). This relatively long pain-free period leads us to hypothesize that this repeated protocol over time could be particularly useful in a subset of patients with drug-resistant $\mathrm{CCH}$. Further ad hoc research is needed to confirm this hypothesis.

Furthermore, we confirm the low frequency and severity of adverse events. This certainly supports the choice of local against oral steroid therapy, especially when other cardiovascular or gastric comorbidities are present.

The absence of a control group is a limit of our study, as clusters may change following their natural course and independently of therapeutic approaches. However, in our sample the short duration of $\mathrm{CH}$ since onset positively correlates with a good response to GON blockade. Thus, a significant impact of cluster early termination on rate of response is unconvincing, though not totally excludable.

The observational design of our study obviously makes it susceptible to placebo effect, which has also been described in $\mathrm{CH}$ patients [20]. However, the long persistence of the response makes it unlikely. A clear point of strength is the consecutive recruitment of patients, which grants the representativeness of the group studied for the larger population of patients followed at our Center. 


\section{Conclusions}

Repeated greater occipital nerve injections of $60 \mathrm{mg}$ methylprednisolone, performed on alternate days, is an effective therapy in $\mathrm{CH}$. We observed a complete response in near half of $\mathrm{ECH}$ and in a third of $\mathrm{CCH}$ for at least of 1 month after the last injection. This protocol seems particularly useful in the treatment of $\mathrm{CCH}$, leading to a 3-month pain-free period in responder patients. Adverse effects are mild and support its use as first choice compared to oral therapies. More studies are needed to compare these results to other types, dosages and timing of local therapies.

Supplementary Information The online version contains supplementary material available at https://doi.org/10.1007/s00415-021-10884-0.

Author contributions EM had a major role in the acquisition of data and drafted the manuscript for intellectual content; GMA contributed to design and conceptualized study and drafted the manuscript for intellectual content; VF, DM and AS contributed in data acquisition; $\mathrm{CZ}$ performed data analysis; $\mathrm{PC}$ made critical review, commentary or revision; SC and GP are responsible for ideas and formulation of research goals and aims.

Funding We declare no funding.

Availability of data and materials All data generated or analysed during this study are included in this published article and its supplementary information files.

\section{Declarations}

Conflicts of interest The authors declare that they have no competing interests.

Ethics approval and consent to participate The study was approved by the Local Ethics Committee of the health service of Bologna (Comitato Etico Indipendente Area Vasta Emilia Centro: CE 17130) and all patients gave their written informed consent to study participation.

Consent for publication All participants gave informed consent for publication of their personal anonymized data.

Open Access This article is licensed under a Creative Commons Attribution 4.0 International License, which permits use, sharing, adaptation, distribution and reproduction in any medium or format, as long as you give appropriate credit to the original author(s) and the source, provide a link to the Creative Commons licence, and indicate if changes were made. The images or other third party material in this article are included in the article's Creative Commons licence, unless indicated otherwise in a credit line to the material. If material is not included in the article's Creative Commons licence and your intended use is not permitted by statutory regulation or exceeds the permitted use, you will need to obtain permission directly from the copyright holder. To view a copy of this licence, visit http://creativecommons.org/licenses/by/4.0/.

\section{References}

1. Headache Classification Committee of the International Headache Society (IHS) (2018) The international classification of headache disorders, 3rd edition. Cephalalgia 38:1-211

2. Peres MF, Stiles MA, Siow HC et al (2002) Greater occipital nerve blockade for cluster headache. Cephalalgia 22:520-522

3. Ekbom K, Solomon S (2000) Management of cluster headaches. In: Olesen J, Tfelt Hansen P, Welch KMA (eds) The headaches, 2nd edn. Lippincott Williams \& Wilkins, Philadelphia, pp 731-740

4. Robbins MS, Starling AJ, Pringsheim TM et al (2016) Treatment of cluster headache: the American Headache Society evidencebased guidelines. Headache 56:1093-1106

5. Ornello R, Lambru G, Caponnetto V, Frattale I et al (2020) Efficacy and safety of greater occipital nerve block for the treatment of cluster headache: a systematic review and meta-analysis. Expert Rev Neurother 20(11):1157-1167

6. Leroux E, Valade D, Taifas I et al (2011) Suboccipital steroid injections for transitional treatment of patients with more than two cluster headache attacks per day: a randomized, double-blind, placebo-controlled trial. Lancet Neurol 10:891-897

7. Natsis K, Baraliakos X, Appell HJ et al (2006) The course of the greater occipital nerve in the suboccipital region: a proposal for setting landmarks for local anesthesia in patients with occipital neuralgia. Clin Anat 19:332-336

8. Zung WW (1971) A rating instrument for anxiety disorders. Psychosomatics 12(6):371-379

9. Beck AT, Steer RA, Brown G (1996) Beck depression inventoryII. APA PsycTests. https://doi.org/10.1037/t00742-000

10. Ustun TB, Kostanjesek N, Chatterji S, Rehm J, World Health Organization. Measuring health and disability: manual for WHO Disability Assessment Schedule (WHODAS 2.0)!' ISBN: 9789241547598

11. Bigo A, Delrieu F, Bousser MG (1989) Treatment of vascular pain of the face by methylprednisolone injection into the area of the greater occipital nerve: 16 cases. Rev Neurol (Paris) 145:160-162

12. Afridi SK, Shields KG, Bhola R et al (2006) Greater occipital nerve injection in primary headache syndromes-prolonged effects from a single injection. Pain 122:126-129

13. Gantenbein AR, Lutz NJ, Riederer F et al (2013) Efficacy and safety of 121 injections of the greater occipital nerve in episodic and chronic cluster headache. Cephalalgia 32:630-634

14. Gaul C, Roguski J, Dresler T et al (2017) Efficacy and safety of a single occipital nerve blockade in episodic and chronic cluster headache: a prospective observational study. Cephalalgia 37(9):873-880

15. Gönen M, Balgetir F, Aytaç E et al (2019) Suboccipital steroid injection alone as a preventive treatment for cluster headache. $\mathrm{J}$ Clin Neurosci 68:140-145

16. Lambru G, Abu Bakar N, Stahlhut L et al (2014) Greater occipital nerve blocks in chronic cluster headache: a prospective open-label study. Eur J Neurol 21(2):338-343

17. Rozen TD (2019) High-volume anesthetic suboccipital nerve blocks for treatment refractory chronic cluster headache with long-term efficacy data: an observational case series study. Headache 59:56-62

18. Ambrosini A, Vandenheede M, Rossi P et al (2005) Suboccipital injection with a mixture of rapid- and long-acting steroids in cluster headache: a double blind placebo-controlled study. Pain 118:92-96

19. Kerr FWL (1961) A mechanism to account for frontal headache in cases of posterior fossa tumour. J Neurosurg 18:605-609

20. Nilsson Remahl AIM, Laudon Meyer E, Cordonnier C, Goadsby PJ (2003) Placebo response in cluster headache trials: a review. Cephalalgia 23:504-510 